\title{
A Random Sampling O(n) Force-calculation Algorithm for Graph Layouts
}

\author{
R. Gove (D), Two Six Labs, USA
}

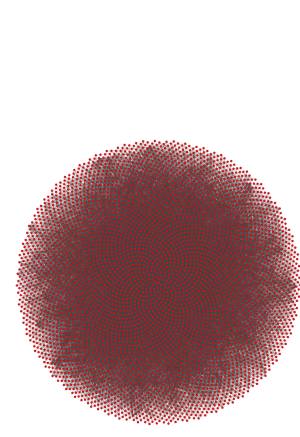

(a)

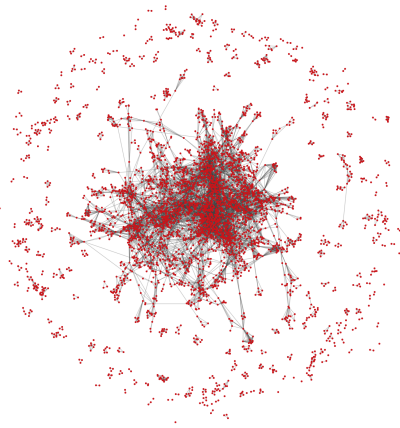

(b)

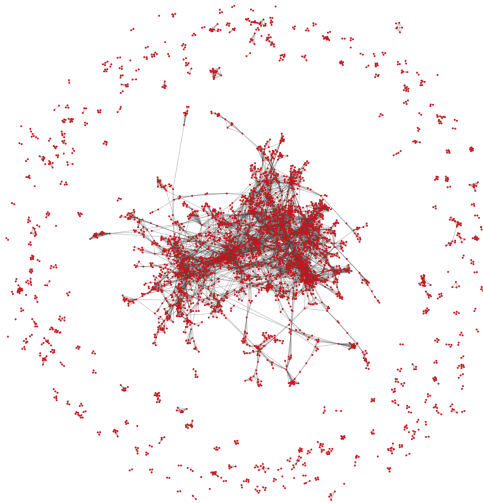

(c)

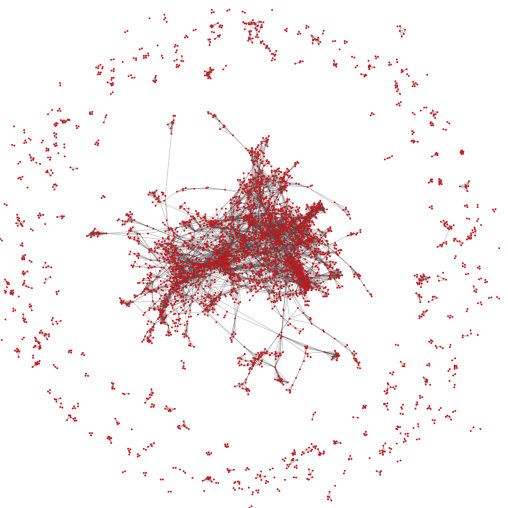

(d)

Figure 1: Iterative layouts of the TVCG collaboration graph (4,345 vertices and 11,732 edges) created using the random vertex sampling layout algorithm, which runs in $O(|V|+|E|)$ time. This figure shows the initial vertex positions $(a)$, and the layout after $20(b), 100(c)$, and 300 (d) iterations of the algorithm. This took about 7.0 seconds, whereas a Barnes-Hut based algorithm took about 17.7 seconds.

\begin{abstract}
This paper proposes a linear-time repulsive-force-calculation algorithm with sub-linear auxiliary space requirements, achieving an asymptotic improvement over the Barnes-Hut and Fast Multipole Method force-calculation algorithms. The algorithm, named random vertex sampling $(R V S)$, achieves its speed by updating a random sample of vertices at each iteration, each with a random sample of repulsive forces. This paper also proposes a combination algorithm that uses RVS to derive an initial layout and then applies Barnes-Hut to refine the layout. An evaluation of RVS and the combination algorithm compares their speed and quality on 109 graphs against a Barnes-Hut layout algorithm. The RVS algorithm performs up to 6.1 times faster on the tested graphs while maintaining comparable layout quality. The combination algorithm also performs faster than Barnes-Hut, but produces layouts that are more symmetric than using RVS alone. Data and code: https://osf.io/nb 7m8/
\end{abstract}

CCS Concepts

- Human-centered computing $\rightarrow$ Graph drawings; Empirical studies in visualization

\section{Introduction}

Graphs, denoted $G=(V, E)$, are important for analyzing data such as social networks or Internet traffic. Straight-line drawings are a common way to visualize graphs. Ideally, graph layout algorithms will be fast and simple to implement, but also produce high quality layouts. However, current algorithms are either slower than linear time [QE00, FR91, JVHB14, Hu05], require complicated algorithms or mathematical operations [HJ05b, GK02], or do not always produce high quality layouts [KCH02, ÇMIBR06, BP06]. For example, force-directed layout algorithms are widely considered to be conceptually simple [FR91, Kob16, ADLM15, LWZ15], they have many usability advantages over other layouts [VR08,DLF*09, Hua07, PSD09], and they have been widely adopted [JVHB14, GN99, Bel, BHJ09, HCL05, Hee08, BH09, BOH11] unlike some other layout algorithms that use more complicated algorithms or mathematical operations (a recent survey identified difficulty in reproducing research as the most common reason for not adopting research [Won17]). However, current force-directed algorithms run in $O(|V| \log |V|+|E|)$ time and require $O(|V| \log |V|+|E|)$ space. 
To address the lack of fast, simple, and high quality graph layout algorithms, this paper presents random vertex sampling (RVS), a $O(|V|)$ time and $O\left(|V|^{3 / 4}\right)$ auxiliary space open source (https://github.com/twosixlabs/ d3-force-sampled) force-directed algorithm that uses random sampling to compute repulsive forces. It is an alternative to the standard Barnes-Hut (BH) [BH86] and the Fast Multipole Method (FMM) [AGPS98, Gre87, HJ04] algorithms and takes a completely different approach. Whereas BH and FMM run in $O(|V| \log |V|)$ time with $O(|V| \log |V|)$ space and are based on trees and aggregations, in contrast, RVS foregoes trees and aggregations. Instead, RVS works by updating a $|V|^{3 / 4}$ random subset of vertices at each iteration, each with a $|V|^{1 / 4}$ random subset of repulsive forces. This paper also proposes RVS\&BH, a combination algorithm that uses RVS to derive an initial layout and BH to refine the layout.

This paper evaluates the RVS and RVS\&BH algorithms on 109 small and large graphs. Compared to a force directed algorithm using the BH approximation, RVS runs up to 6.1 times faster on the test graphs. There is no statistically significant difference in graph readability metrics between the three algorithms, suggesting that the algorithms produce layouts of equivalent quality. Qualitatively, for some graphs, layouts from the algorithms appear to be identical for practical purposes, but on other graphs the RVS layout appears less symmetric. However, the combination algorithm of RVS and $\mathrm{BH}$ runs up to 4.8 times faster than $\mathrm{BH}$, and produces better symmetry than using RVS alone.

This paper contributes (1) a novel random sampling repulsive force algorithm that runs in $O(|V|)$ time with $O\left(|V|^{3 / 4}\right)$ auxiliary space, (2) a novel repulsive force algorithm that combines RVS and $\mathrm{BH}$, (3) an evaluation of the RVS algorithm's runtime showing that it performs several times faster than a $\mathrm{BH}$ based algorithm, and (4) an evaluation showing that the RVS algorithm produces layouts with similar quality to a BH based algorithm.

\section{Related Work}

Many types of graph layouts exist (one classification is by forcebased, constraint-based, multi-scale, layered, and non-standard layouts [VKS*11]). This section discusses related work on forcedirected, approximation, or random sampling techniques. ("Forcedirected" can refer to either stress-minimization algorithms or "spring-electric" algorithms that model vertices as charged particles and edges as springs. This paper uses the latter meaning.)

Force-Directed Graph Layouts The history of force-directed graph layouts can be traced back to Tutte [Tut63]. Eades [Ead84] proposed modeling edges with springs and using repulsive forces between vertices to repel each other using a naïve brute force $n$-body simulation where each iteration is $O\left(|V|^{2}+|E|\right)$ time. Fruchterman and Reingold [FR91] refined this idea by adding the notion of "temperature" that reduces the amount of vertex displacement as the simulation iterates.

Although some force-directed algorithms can be slow, they have many benefits: (1) they make no assumption that graphs are connected, in contrast with layout algorithms based on dimensionality reduction that often implicitly assume that graphs are connected; (2) force-directed algorithms are widely considered to be a conceptually simple way to create graph layouts [FR91, Kob16, ADLM15, LWZ15]; and (3), several evaluations have shown that force-directed layouts have advantages over other layouts for analysis tasks [VR08, DLF*09, Hua07, PSD09].

Approximate Repulsive Force Calculations To speed up the $O\left(|V|^{2}\right)$ repulsion calculations, Fruchterman and Reingold [FR91] experimented with partitioning the space into a grid, but the worst case running time was still quadratic. Instead of using a grid, another approach is to cluster vertices [HA15]. Using this approach, two repulsive forces are calculated on each vertex $v$ : (1) the pairwise repulsive force between each vertex within the same cluster, and (2) the repulsive force from the barycenters of all clusters than do not contain $v$. Vertices are assigned to their nearest clusters, which takes $O(c|V|)$ time for $c$ clusters. If each cluster has the same number of vertices, naïve pairwise repulsive force calculation takes $O\left(c(|V| / c)^{2}\right)=O\left(|V|^{2} / c\right)$ time. If $c$ is constant, then vertex assignment is linear time, but force calculation is quadratic time; on the other hand, if $c=\sqrt{|V|}$, then vertex assignment and force calculation are both $O(|V| \sqrt{|V|}$ time. Applying an approximation method like Barnes-Hut to the pairwise repulsive force calculation can improve the runtime, but either vertex assignment or repulsive force calculation will always be slower than $O(|V|)$.

In the 1980s and 1990s, researchers proposed the BarnesHut [BH86], Fast Multipole Method [Gre87], and Well-Separated Pair Decomposition [CK95] algorithms for approximating repulsive force calculations. These methods use a $O(|V| \log |V|)$ runtime preprocessing step to build a spatial tree of the vertex positions [AGPS98], and then they use the spatial tree to compute aggregate forces. Barnes-Hut and the Well-Separated Pair Decomposition compute forces in $O(|V| \log |V|)$ time and the Fast Multipole Method computes forces in $O(|V|)$ time, but all are overall $O(|V| \log |V|)$ runtime because of the tree preprocessing step. Researchers applied these tree-aggregation force-approximation algorithms to computing graph layouts [QE00, HJ05b, LWZ16]. Updating these approximation methods' spatial trees less often is a way to speed up their runtime without affecting layout quality, but their asymptotic complexity is still $O(|V| \log |V|)$ [LWZ16, Gov18b].

The Barnes-Hut approximation is very popular for graph layouts-many algorithms use it, such as ForceAtlas2 [JVHB14], MARS [KHKS12], and Hu's multi-scale algorithm [Hu05], along with popular software packages such as GraphViz [GN99], JavaScript InfoVis Toolkit [Bel], Gephi [BHJ09], Prefuse [HCL05], Flare [Hee08], Protovis [BH09], and D3 [BOH11]. The Fast Multipole Method is often regarded as difficult to implement [Dar00, VA10], which might explain why it has not been adopted in as many graph layout software packages.

Table 1 provides an overview of the time and space complexity of these repulsive-force calculation algorithms.

This paper proposes combining random vertex sampling (RVS) with Barnes-Hut $(\mathrm{BH})$ by first using RVS to derive good initial positions, and then using $\mathrm{BH}$ to refine the layout. This is similar to multi-scale layout algorithms [Hu05, HJ05b, AMA07] that use graph filtration to derive aggregated versions of the original graph, then compute a layout for the aggregated graphs, and finally use the layouts of the aggregated graphs to derive initial positions for 
Table 1: Summary of repulsive-force calculation algorithms. Initialization time is the time required at each iteration of the algorithm before forces can be calculated (e.g. to prepare data structures or assign clusters). Force calculation time is the time required to compute repulsive forces using the specified method. Auxiliary space is the extra space needed by the method (e.g. to store spatial trees or cluster assignments).

\begin{tabular}{|c|c|c|c|c|c|}
\hline Method & $\begin{array}{l}\text { Initialization } \\
\text { time }\end{array}$ & $\begin{array}{c}\text { Force } \\
\text { calculation time }\end{array}$ & $\begin{array}{l}\text { Auxiliary } \\
\text { space }\end{array}$ & Notes & Reference \\
\hline Naïve & $O(1)$ & $O\left(|V|^{2}\right)$ & $O(1)$ & & [Ead84] [FR91] \\
\hline Grid & $O(|V|)$ & $O\left(|V|^{2}\right)$ & $O(|V|)$ & & [FR91] \\
\hline Cluster & $O(c|V|)$ & $O\left(|V|^{2} / c\right)$ & $O(|V|)$ & $c$ is the number of clusters & [HA15] \\
\hline $\begin{array}{c}\text { Tree } \\
\text { aggregation }\end{array}$ & $O(|V| \log |V|)$ & $\begin{array}{c}O(|V|) \\
\text { or } O(|V| \log |V|)\end{array}$ & $O(|V| \log |V|)$ & $\begin{array}{l}\text { FMM is } O(|V|) \text { force } \\
\text { calculation time; BH and } \\
\text { WSPD are } O(|V| \log |V|)\end{array}$ & $\begin{array}{c}\text { [BH86] [Gre87] } \\
{[\mathrm{CK} 95][\mathrm{AGPS98}]} \\
{[\mathrm{QE00]}[\mathrm{HJ} 05 \mathrm{~b}]}\end{array}$ \\
\hline $\begin{array}{l}\text { Random } \\
\text { sampling }\end{array}$ & $O\left(|V|^{3 / 4}\right)$ & $O(|V|)$ & $O\left(|V|^{3 / 4}\right)$ & & $\begin{array}{c}\text { Proposed in } \\
\text { this paper }\end{array}$ \\
\hline
\end{tabular}

the vertices in the unaggregated graphs. TopoLayout [AMA07] in particular uses different layout algorithms tuned for each subgraph. However, the RVS\&BH combination algorithm is different because it does not rely on multi-scale algorithms, and it applies both RVS and $\mathrm{BH}$ globally across the entire graph instead of selectively using different layout algorithms on different subgraphs. Furthermore, although multi-scale graph layout algorithms can produce very good layouts, they do not appear to support composing forces and adding constraints as easily as single-level force-directed algorithms. This is because the merging algorithms may merge two vertices of different types into a single parent vertex, which can confound constraints that require the two vertex types to be treated separately. Therefore it is useful to develop and evaluate general purpose forcedirected layout algorithms independently of multi-scale algorithms.

A full survey of other categories of layout algorithms is beyond the scope of this paper (see Kobourov [Kob16] and von Landesberger et al. [VKS*11] for useful surveys). Some important notes are that many of these algorithms, such as GRIP [GK02], are still slower than linear time. Algorithms like SSDE [ÇMIBR06] sample vertices to compute graph theoretic distances to use in spectral decomposition. HDE [HK02], ACE [KCH02], SSDE [ÇMIBR06], and Pivot MDS [BP06] are fast (e.g. HDE, SSDE, and Pivot MDS are linear time), but they have known issues with preserving neighborhoods [KRM*17] and producing good layouts for tree-like and other non-mesh-like graphs [KRM*17,ÇMIBR06,HJ07]. These algorithms have not been widely adopted, unlike force-directed algorithms that enjoy widespread adoption in many software packages.

Random Sampling Proxy graphs are representatives of larger graphs that are derived through sampling, filtering, or deriving a structural skeleton such as a spanning tree [ENH17, Zha15, NHEM17, WCA*17, ZZC*17, RC05]. Therefore, these proxy graphs are missing some vertices and/or edges, and their visualizations will not show all the data. In contrast, this paper visualizes all the vertices and edges of a graph, and only uses random sampling to reduce the number of calculations when positioning vertices.

To produce proxy graphs, random edge sampling performs as well as spectral sparsification [ENH17], a more complicated technique. Eades et al. make the case that random edge sampling can be better because of its speed and simplicity, indicating that a random sampling strategy can be useful for graph calculations.

Chalmers [Cha96] used a random sampling spring-electric model for multidimensional scaling, where each vertex had a constant-sized list of "neighbors" that were used to compute repulsion forces on each vertex. At each iteration, when updating a vertex, a constant-sized list of randomly chosen vertices were also used to compute repulsive forces on the current vertex. This use of "neighbors" and random sampling is similar to the random vertex sampling used in this paper, but the randomly chosen vertices are of fixed size. In addition, Chalmers does not randomly choose a subset of vertices to update at each iteration. Chalmers' technique does not appear to have been applied to computing graph layouts.

Morrison et al. [MRC03] use a strategy of subsampling $\sqrt{n}$ data points from a dataset of size $n$, using the Chalmers technique to position the $\sqrt{n}$ data points, and then positioning the remaining data points relative to the $\sqrt{n}$ data points in a constant number of iterations. This is similar to the random sampling strategy explored in this paper, except that this paper uses a sliding window approach, random sampling to select vertices to compute repulsion, and does not use a relative position step.

\section{RVS Algorithm Description}

Random Vertex Sampling (RVS) is a plugin for v4 and v5 of the D3 JavaScript visualization library [BOH11]. The RVS plugin uses D3's existing force-directed graph layout algorithm, but replaces the Barnes-Hut $(\mathrm{BH})$ vertex repulsion algorithm with a new RVS repulsion component. This section describes some components of the D3 force-directed layout as well as the RVS plugin.

\subsection{Initialization}

The layout algorithm operates on the vertex and edge sets $V$ and $E$, which are stored as arrays. The algorithm executes an initialization step before it calculates the attractive and repulsive forces. This initializes the vertex positions in a phyllotaxis arrangement resembling a disk shape, where vertices closer to the beginning of the vertex array are placed closer to the center (see Figure 1a). The $x$ and $y$ velocities $u_{v_{x}}$ and $u_{v_{y}}$ of each vertex $u$ are initialized to $0 . \alpha$ is 
a "cooling" parameter (also called a temperature parameter), which is initialized to 1 . An array $A$ is created that contains each integer from 1 to $|V|$, to be used by the FisherYates and RVS procedures.

\subsection{Random Vertex Sampling Repulsion}

The RVS repulsive-force algorithm contains two components: Computing repulsive forces using randomly selected vertices, and computing repulsive forces using a fixed-sized set of vertices.

The core of the RVS algorithm is as follows: at each iteration of the algorithm, update the velocities of a subset of vertices $U \subseteq V$. Then for each updating vertex $u \in U$, select a random set of vertices $R \subseteq V$ to compute repulsive forces on $u$. If $U$ and $R$ can be found in $O(|U|)$ and $O(|R|)$ time, and if $|U|=|V|^{\phi}$ and $|R|=|V|^{1-\phi}$ for $0<$ $\phi<1$, then the repulsive forces can be calculated in $O(|U| \cdot|R|)=$ $O\left(|V|^{\phi} \cdot|V|^{1-\phi}\right)=O(|V|)$ time. Furthermore, this algorithm only requires $O\left(|V|^{\phi}+|V|^{1-\phi}\right)$ auxiliary space, which is used to store $U$ and $R$. This RVS procedure is described in Algorithm 1. The subset $R$ is created using a modification of the Fisher-Yates shuffle (Algorithm 2). Whereas the canonical Fisher-Yates shuffle returns a random permutation of $n$ numbers in $O(n)$ time, this algorithm returns $k$ integers in the range $[1, n]$ and runs in $O(k)$ time.
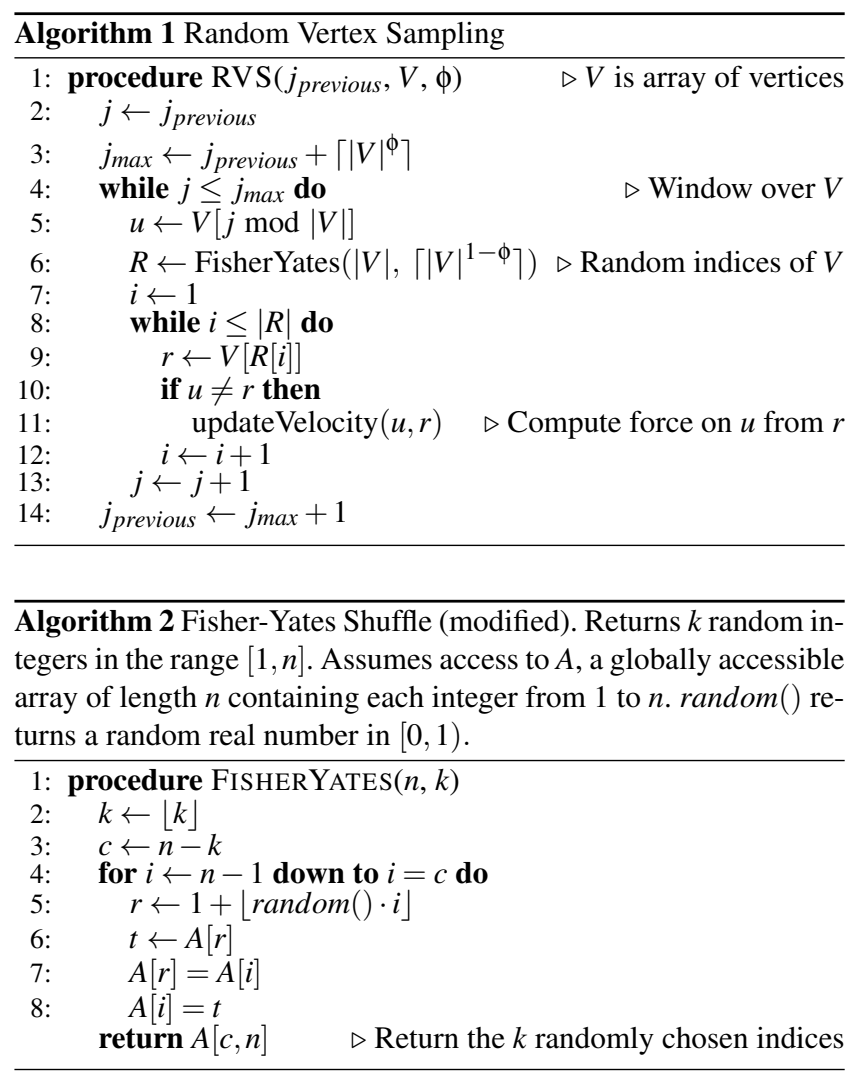

RVS uses a sliding window over the array of vertices to create the subset $U$. Each time the RVS procedure is called, it receives the index $j$ of the last vertex updated in the previous iteration, thereby shifting the window forward (this paper uses 1-based indexing, so the first time RVS is called, $j_{\text {previous }}=1$ ). The RVS procedure is called once per iteration of the algorithm.

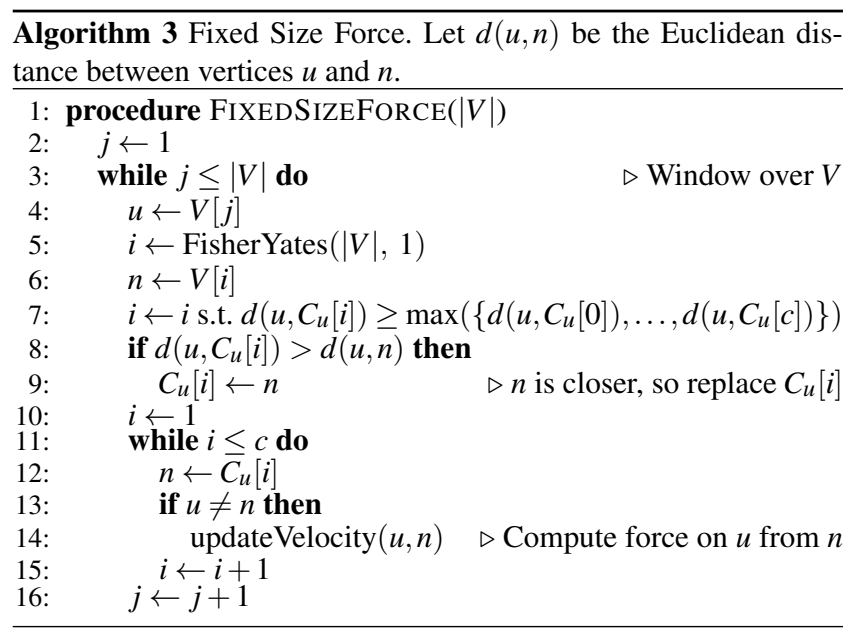

The updateVelocity $(u, r)$ procedure uses these equations to update vertex $u$ 's velocity using the repulsive force from vertex $r$ :

$$
\begin{aligned}
& u_{v_{x}}=u_{v_{x}}+\frac{\delta_{x} \cdot r_{\text {strength }} \cdot q_{\text {mult }} \cdot \alpha}{\delta_{x}^{2}+\delta_{y}^{2}} \\
& u_{v_{y}}=u_{v_{y}}+\frac{\delta_{y} \cdot r_{\text {strength }} \cdot q_{\text {mult }} \cdot \alpha}{\delta_{x}^{2}+\delta_{y}^{2}}
\end{aligned}
$$

where $\delta_{x}=r_{x}-u_{x}, \delta_{y}=r_{y}-u_{y}, r_{\text {strength }}$ is the charge repulsive strength of vertex $r$ (default is -30 ), and $q_{\text {mult }}$ is a charge multiplier to compensate for computing repulsive forces on only a small subset of vertices. If $0 \leq|V| \leq 100$ then $q_{\text {mult }}=1$, if $100<|V| \leq 200$ then $q_{\text {mult }}=3$, and if $|V|>200$ then $q_{\text {mult }}=\sqrt{|V|}$, which was determined by experience developing the algorithm. Experience also indicated that $\phi=3 / 4$ performed better than other values such as $1 / 2$ or $2 / 3$. Especially for small graphs, updating vertices more often with less accurate repulsive forces is better than updating less often with more accurate repulsive forces. For larger values of $\phi$ the size of $R$ becomes too small to be useful.

Each vertex $u$ has a fixed-sized set $C_{u}$ of $c=\min (15,|V|)$ vertices that are initially chosen randomly (derived from experience developing the algorithm). $C_{u}$ is updated at each iteration of the algorithm, choosing a new random vertex $n$ and replacing the vertex in $C_{u}$ that is farthest from $u$ if $n$ is closer to $u$. Each iteration of the algorithm computes repulsive forces on each vertex $u$ using it's set of vertices $C_{u}$ and the same equations as above. See Algorithm 3 . Because $c$ is constant, computing forces runs in $O(c|V|)=O(|V|)$ time, similar to the "neighbors" list used by Chalmers [Cha96]. This serves two purposes: First, at the beginning of the algorithm, the forces from each vertex's $C_{u}$ help to spread out the graph's components from their initial positions where they are close together. Second, near the end of the layout, the forces from each vertex's $C_{u}$ help large components from being becoming too compact if the layout algorithm uses a gravitational force to attract vertices toward the layout's center. Experience developing the algorithm indicated that neither using forces from $C_{u}$ nor RVS alone was enough to produce good quality layouts for large graphs; combining both produced better layouts than either one individually.

Note that if $|V|$ is approximately 8 billion, then it will require 
$|V|^{1 / 4} \approx 300$ iterations to compute repulsive forces on each vertex. However, this is an extraordinarily large graph. The largest graph studied in this paper has 143,437 vertices, requiring about 19 iterations to compute repulsive forces on each vertex. 10,000,000 vertices requires 56 iterations. Therefore, this is unlikely to be a problem in practice for three reasons: $f(V)=|V|^{1 / 4}$ grows very slowly, $|V|^{1 / 4}$ only becomes large for uncommonly large graphs, and RVS uses each vertex's $C_{u}$ to compute forces at every iteration.

\subsection{Updating Variables}

After calculating all relevant forces, the algorithm updates the $x$ and $y$ velocity of $u$ using the velocity decay constant $V_{d}$ :

$$
\begin{aligned}
& u_{v_{x}}=u_{v_{x}} \cdot\left(1-V_{d}\right) \\
& u_{v_{y}}=u_{v_{y}} \cdot\left(1-V_{d}\right)
\end{aligned}
$$

The algorithm then updates the $x$ and $y$ position for each vertex $u$ using $u$ 's velocity:

$$
\begin{aligned}
& u_{x}=u_{x}+u_{v_{x}} \\
& u_{y}=u_{y}+u_{v_{y}}
\end{aligned}
$$

Note that RVS can achieve good results because it explicitly stores the velocity and then uses and updates it during the next iteration, instead of recalculating the repulsive velocity at every iteration based only on the current vertex positions. This means that even if a vertex is not selected to be updated in the current iteration, it may still have some residual repulsive velocity from a previous iteration.

Finally, before each iteration ends, the algorithm updates $\alpha$ :

$$
\alpha=\alpha+\left(\alpha_{t}-\alpha\right) \cdot \alpha_{d}
$$

where $\alpha_{t}$ is the target $\alpha$ value (default is 0 ), and $\alpha_{d}$ is the alpha decay rate. This paper uses $\alpha_{d}=1-\alpha_{m}^{1 / 300}$, which results in 300 iterations of the algorithm before $\alpha$ reaches $\alpha_{m}$, the minimum $\alpha$, thereby triggering the algorithm to stop.

\section{Combining RVS and BH}

Experience showed that Random Vertex Sampling (RVS) can produce layouts that are less symmetric than Barnes-Hut (BH). This is most visible in graph neighborhoods that resemble trees, such as the eva benchmark graph of corporate relationships. An alternate layout algorithm is to combine RVS and BH: First run several iterations of RVS to derive good initial positions, and then run a few iterations of $\mathrm{BH}$ to refine the layout. Experience developing the algorithm showed that 10 iterations of $\mathrm{BH}$ can be enough to improve the layout (see Figure 2 for an example of running 300, 290, 280, and 270 iterations of RVS followed by 0, 10, 20, and 30 iterations of $\mathrm{BH}$, respectively). This leads to the proposed combination algorithm: First run 290 iterations of RVS, and then use that result as the initial positions for 10 iterations of $\mathrm{BH}$. Because the combined algorithm uses $\mathrm{BH}$, it runs in $O(|V| \log |V|)$ time.

\section{Runtime Performance Evaluation}

To evaluate runtime performance, this evaluation compares three graph layout algorithms using random vertex sampling (RVS), the Barnes-Hut (BH) approximation, and a combination of RVS and

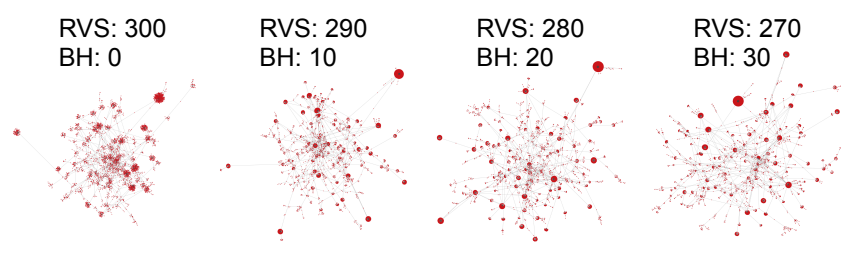

Figure 2: Four layouts of the eva graph. On the left is a layout that only uses RVS. The other layouts combine RVS and BH by use varying numbers of iterations of RVS to generate initial positions followed by a few iterations of BH to improve the layout. Only 10 iterations of $\mathrm{BH}$ are enough to substantially improve the layout.

BH (RVS\&BH) described in section 4. (This evaluation does not include multi-scale layout algorithms for reasons of experimental validity and because force-directed algorithms can have uses independent of multi-scale algorithms, as discussed in section 2.) All algorithms incorporate the edge and gravitational forces using the default values and initial parameters defined above. For BH, velocity decay $V_{d}=0.4$, and $V_{d}=0.2$ for RVS. D3's default is $v_{d}=0.4$, but experience developing RVS indicated that 0.2 results in layouts with similar spacing as $\mathrm{BH}$ with $V_{d}=0.4$. Each algorithm terminates after 300 iterations.

Table 2: Summary of the 109 evaluation graphs: The number of graphs in each class, and the vertex $(|V|)$, edge $(|E|)$, normalized components $(C)$, and density $(D)$ ranges rounded to two decimal places (i.e. 0.00 indicates a small value rounded to 0.00 , and $0 \mathrm{in}$ dicates the value is exactly 0 ). The normalized component metric for a graph is calculated by subtracting 1 from the number of components and then dividing by $|V|-1$.

\begin{tabular}{lrcccc} 
Class & $\#$ & $|V|$ & $|E|$ & $C$ & $D$ \\
\hline \hline \multicolumn{7}{c}{ Small graphs } \\
\hline art. & 12 & $250-1,000$ & $741-4,000$ & 0 & $0.01-0.03$ \\
char. & 14 & $18-138$ & $41-493$ & $0-0.05$ & $0.05-0.27$ \\
ego & 9 & $53-787$ & $198-30,772$ & 0 & $0.04-0.18$ \\
geo. & 14 & $4-20$ & $6-32$ & 0 & $0.16-1$ \\
soft. & 8 & $128-716$ & $310-3,579$ & $0.00-0.05$ & $0.00-0.04$ \\
sub. & 15 & $82-433$ & $85-475$ & 0 & $0.01-0.03$ \\
\hline \multicolumn{7}{c}{ Large graphs } \\
bench. 21 & $1,005-143,437$ & $2,596-2,031,480$ & $0.00-0.01$ & $0.00-0.01$ \\
collab. & 12 & $1,001-20,046$ & $2,627-198,110$ & $0.02-0.28$ & $0.00-0.01$ \\
ego & 1 & 1,035 & 27,783 & 0 \\
soft. & 3 & $1,323-2,956$ & $4,809-10,845$ & $0.01-0.03$ & $0.00-0.00$ \\
\hline \multicolumn{7}{c}{}
\end{tabular}

This evaluation uses 109 common graphs from network science and graph algorithm research (note: many graphs from older research are no longer available online). The graphs were grouped by size: 72 graphs with 1,000 or fewer vertices are in the "small" graphs group, and 37 graphs with more than 1,000 vertices are in the "large" graphs group. There is no strict definition of small or large graphs; however, von Landesberger et al. [VKS*11] note that calling a graph "large" typically means it has more than 1,000 vertices. Also, many older graph layout algorithm papers produce layouts on graphs with less than 1,000 vertices [Tut63, FR91, Ead84, DH96, HS95, FLM95], while many graph layout algorithm pa- 
pers that explicitly address scalability evaluate their algorithms on graphs with about 1,000 vertices or more [MMM08, HK02, $\mathrm{KCH} 02$, BP06, ÇMIBR06, QE00, KHKS12, Hu05, HJ05a].

The large and small groups of graphs were further divided into several classes based on each graph's origin or the way the graph was constructed. The artificial graphs were generated using the Barabási-Albert [BA99] and Watts-Strogatz algorithms [WS98]. The character interaction graphs came from CharNet [HMF*17], Stanford GraphBase [Knu94], and The Star Wars Social Network [Gab15]. The ego graphs are Facebook ego graphs [ML12] with the ego vertex re-added to the graph. Geometric graphs are common geometric graphs found in the NetworkX library [HSS08]. The software graphs show dependencies in several software packages [vB11, vB02, vB12, vBB13, vvBB14]. The subway graphs show the subway station connections in subway systems from major cities in 2009 [RKBB12]. The collaboration graphs show co-authorships between researchers in several fields related to computer science, network science, and physics [New01, New06, LKF07, KPS17]. Benchmark graphs mostly come from Network Repository [RA15], the Florida Sparse Matrix Collection [DH11], and Walshaw's Graph Partitioning Archive [Wal00]. The benchmark class contains large $(|V|>1000)$ graphs that are commonly used to evaluate large graph layout algorithms [OKB16, AMA07, KHKS12, Hu05, ÇMIBR06, HJ07, BP06, GK02, KCH02, HK02, $\left.\mathrm{KRM}^{*} 17\right]$. These graphs are mostly large meshes, but they also include a few other graphs such as the California roadway system, the USA powergrid, a 32-bit adder, and a 3D Sierpinksi triangle. The benchmark graphs were chosen so that the reader may compare results in this paper to results in other research papers. See Table 2 for further details on each graph class.

To evaluate the runtime performance, this evaluation computed 20 layouts for each of the 109 graphs using the RVS, BH, and RVS\&BH combination algorithms executed in NodeJS version 9.4.0 on a 2015-model MacBook Pro with a $3.1 \mathrm{GHz}$ Intel Core i7 processor and $16 \mathrm{~GB}$ of RAM. Before initializing the algorithm, the array of vertices was randomly permuted so that each vertex's position in the phyllotaxis arrangement was random. Following system benchmarking best practices, hyperthreading was disabled, the garbage collector was triggered between runs, and the minimum time of the 20 runs was recorded for each graph for each algorithm.

Figure 3 shows the differences in runtime for each graph between the three algorithms (jittering on the horizontal axis reduces overlap between graphs with the same number of vertices). RVS's maximum speedup over BH was approximately 6.1 , and the mean was 2.9. For RVS\&BH, its maximum speedup over $\mathrm{BH}$ was 4.8 , and the mean was 2.8 . We see that RVS outperforms $\mathrm{BH}$ even on most small graphs when $|V|<\left|C_{u}\right|$. This is surprising because in those cases RVS is performing the same number of force calculations as the naïve $O\left(|V|^{2}\right)$ repulsive force algorithm, and it indicates that on small graphs the $\mathrm{BH}$ quadtree creation overhead may be more than BH saves by using the approximation. Furthermore, combining RVS and $\mathrm{BH}$ does not come with a large runtime penalty.

To more carefully evaluate the performance of each algorithm as $|V|$ increases, Figure 4 shows the runtime of each algorithm on three sparse graphs with $10,000,100,000$ and 1,000,000 vertices. The graphs were generated with $|E|=2|V|$ random edges (with-
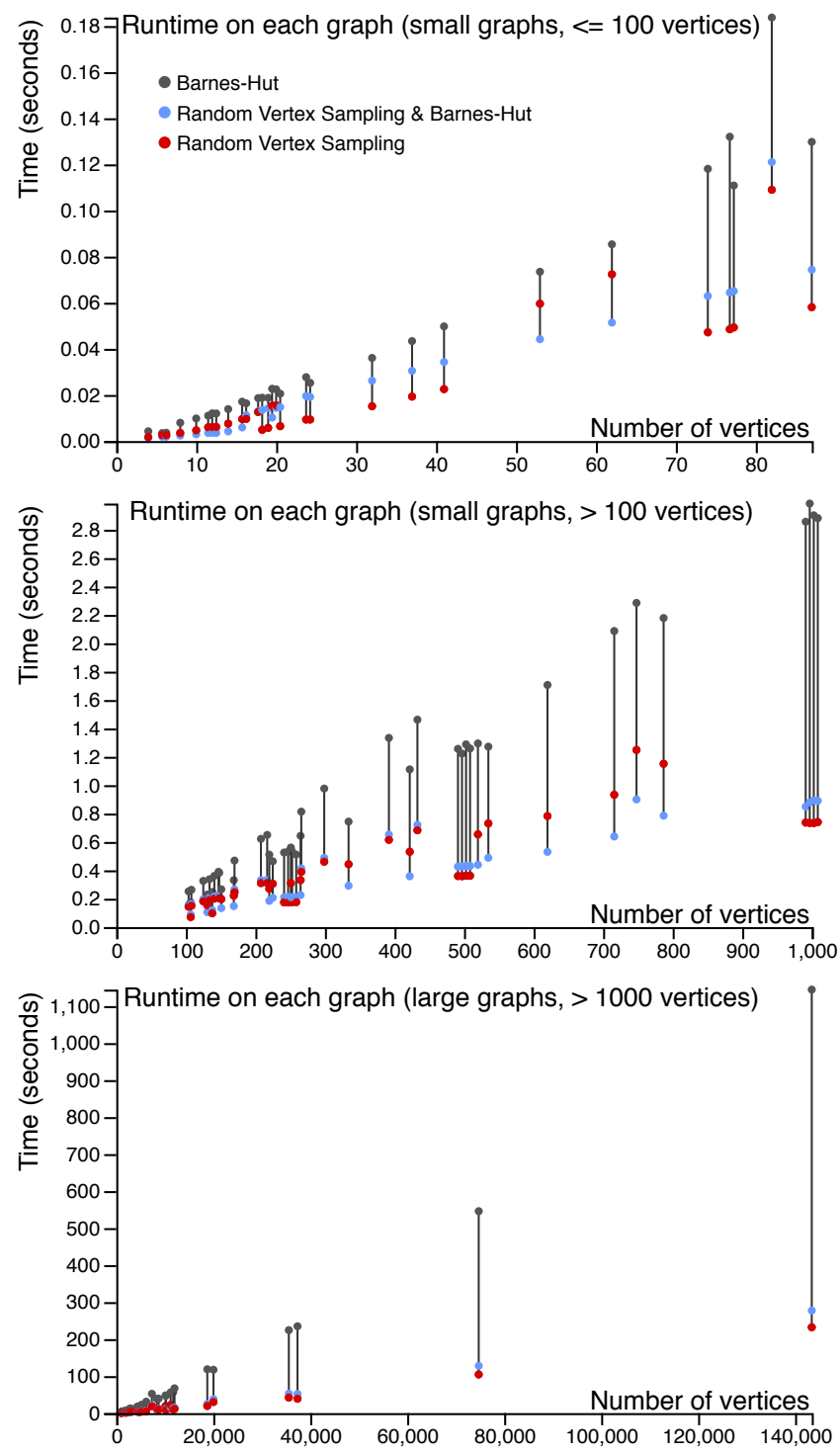

Figure 3: Each corresponding set of red, blue, and black dots represent the runtimes of the RVS, RVS\&BH, and BH layout algorithms on a given graph.

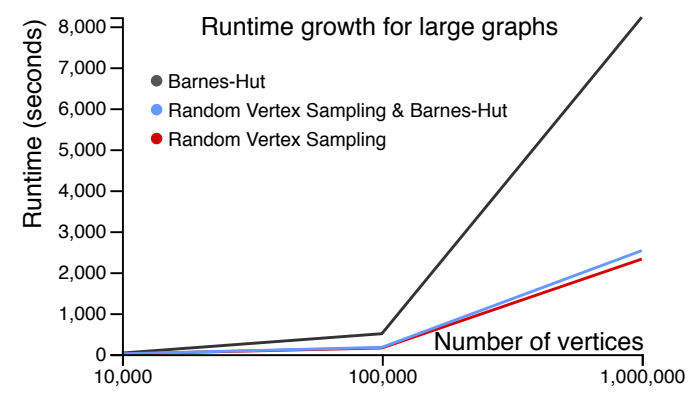

Figure 4: Runtime on large graphs. The $x$-axis is log scale. 
out replacement). Keeping the proportion of edges fixed allows us to more easily understand the change in runtime performance for repulsive force calculation as graphs get larger. The runtime improvement increases as the graphs get larger: RVS is 3.2,3.3, and 3.5 times faster than $\mathrm{BH}$ for the $10,000,100,000$ and $1,000,000$ vertex graphs, respectively. RVS\&BH is $2.9,3.0$, and 3.3 times faster than $\mathrm{BH}$ for the same graphs.

\section{Layout Quality Evaluation}

The layout quality evaluation uses the same layout algorithms, parameters, and graphs used in the runtime evaluation.

\subsection{Readability Metric Evaluation}

This evaluation uses five global graph readability metrics to compare graph layout quality: Edge crossings, edge crossing angle, angular resolution (min), angular resolution (dev), and a shape-based metric [EHNK17]. Greadability.js [Gov18a] was used for all metrics except the shape metric. All metrics produce a continuous value in the range $[0,1]$ where larger numbers are better. See Eades et al. [EHNK17] and Nguyen et al. [NHE17] for a more detailed discussion of shape-based metrics, see Gove [Gov18b] for the angular resolution (dev) equation, and see Dunne et al. [DRSM15] for a detailed discussion of the other readability metrics.

Other readability metrics exist, such as stress or standard deviation of edge length, but this evaluation avoids these readability metrics because they have known issues [EHNK17, GN99, KRM*17]. Namely, non-uniform edge lengths are often necessary to achieve good layouts for real-world graphs [GKN04, KRM ${ }^{*}$ 17]; preserving shortest-path distances, as measured by stress, may not be ideal for producing good layouts [KRM $\left.{ }^{*} 17\right]$; stress is not defined on graphs with more than one component, which often occur in real-world data; and two layouts that convey a graph's structure equally can have different stress values [EHNK17]. In addition, stress and standard deviation of edge length do not have normalized versions that support accurate comparisons between different graphs.

\subsubsection{Definitions}

Edge crossings, denoted $\aleph_{c}$, measures the number of edges that cross in the layout. The metric scales the number of edge crossings, $c$, by an approximate upper bound so that $\aleph_{c} \in[0,1]$.

$$
\aleph_{c}=1-c /\left(\frac{|E|(|E|-1)}{2}-\frac{1}{2} \sum_{u \in V} \operatorname{deg}(u)(\operatorname{deg}(u)-1)\right)
$$

If the denominator is 0 , then $\aleph_{c}=1$.

Edge crossing angle, denoted $\aleph_{c a}$, is the average deviation of the edge crossing angles from the ideal angle $\vartheta$ of 70 degrees.

$$
\aleph_{c a}=1-\frac{\sum_{e \in E} \sum_{e^{\prime} \in c(e)}\left|\vartheta-\theta_{e, e^{\prime}}\right|}{c \vartheta}
$$

where $c(e)$ is the set of edges that intersect $e$, and $\theta_{e, e^{\prime}}$ is the acute angle of the two intersecting edges. If $c \vartheta=0$, then $\aleph_{c}=1$.

Angular resolution ( $\mathrm{min}$ ) is the average deviation of incident edge angles from the ideal minimum angle for each vertex $u$.

$$
\aleph_{r m}=1-\frac{1}{|V|} \sum_{u \in V} \frac{\left|\vartheta_{u}-\theta_{u_{\text {min }}}\right|}{\vartheta_{u}}
$$

Here, $\vartheta_{u}=360 / d(u)$, the degrees between each incident edge if the angles were uniform, and $\theta_{u_{\min }}$ is the smallest measured angle between edges incident on $u$.

Angular resolution ( $\mathrm{dev}$ ) is the average deviation of angles between incident edges on each vertex $u$.

$$
\aleph_{r d}=1-\frac{1}{|V|} \sum_{u \in V, d(u)>1}\left(\frac{1}{2 d(u)-2} \sum_{i}^{d(u)} \frac{\left|\vartheta_{u}-\theta_{i,(i+1)}\right|}{\vartheta_{u}}\right)
$$

Here, $\theta_{i,(i+1)}$ is the angle between adjacent edges $i$ and $i+1$ that are incident on vertex $u$, modulo $d(u)$, the degree of $u$.

Because many of the above readability metrics are derived from user studies on small graphs, and because users' analysis tasks may be different on large graphs than small graphs [EHNK17], it is not clear that the above readability metrics are useful for quantifying the readability of large graphs [EHNK17, KPS14]. To address this, Shape-based metrics are a type of neighborhood metric that attempt to quantify the faithfulness of a graph layout by measuring the similarity between a graph layout and a proximity graph derived from the same graph layout. Nguyen et al. [NHE17] suggest using the degree-sensitive neighborhood graph (dNNG) to address several known issues with other proximity graphs, although there is no consensus on which proximity graph to use. For a 2D layout of a graph $G=(V, E)$, let $P_{V}$ be a point set such that for all $v \in V$ there is a corresponding point $p_{v} \in P_{V}$ where $p_{v}$ is the 2D point of $v$ in the graph layout. Then we compute a new graph $\operatorname{dNNG}\left(P_{V}\right)=\left(P_{V}, P_{E}\right)$ where $P_{V}$ is the vertex set and $P_{E}$ is the edge set, where there is a directed edge $p_{e}=\left(p_{v}, p_{u}\right) \in P_{E}$ connecting $p_{v}$ to $p_{u}$ if $v, u \in V$ and $p_{u}$ is in the $d\left(p_{v}\right)$ nearest points to $p_{v}$ or $p_{v}$ is in the $d\left(p_{u}\right)$ nearest points to $p_{u}$ (measured using Euclidean distance). Let $N(v)$ be the neighborhood of $v$ (i.e. the set of vertices incident on $v$ ). Then the shape metric, $Q_{\mu}$, is defined using the mean Jaccard similarity between the two graphs:

$$
Q_{\mu}\left(G, \mathrm{dNNG}\left(P_{V}\right)\right)=\frac{1}{|V|} \sum_{v \in V} \frac{\left|N(v) \cap N\left(p_{v}\right)\right|}{\left|N(v) \cup N\left(p_{v}\right)\right|}
$$

\subsubsection{Experiment}

This layout quality evaluation uses the same experimental setup as the runtime evaluation and follows recommended best practices [Car14, Dra16]. This evaluation bases its analysis on the median of each graph's readability metrics from 20 runs. Because of the issues using traditional readability metrics on large graphs, this evaluation uses only the shape metric on the group of large graphs, and only the other four readability metrics on the group of small graphs (see subsubsection 6.1.1 for further discussion).

This evaluation uses a within-subjects design, comparing the median readability metrics of each graph between the layout algorithms. Because this is comparing three algorithms across five response variables, the evaluation uses a Bonferroni corrected significance level $\alpha=0.05 / 15=0.00 \overline{3}$, with a confidence interval of $[0.001 \overline{6}, 0.998 \overline{3}]$. Because the readability metrics are bounded in $[0,1]$, and many are not normally distributed, this evaluation uses bootstrapped confidence intervals with 10,000 samples. Effect sizes are sample mean difference. Figure 5 shows the mean and bootstrap confidence intervals of the effect sizes for each readability metric. All of the confidence intervals touch or cross 0 . Therefore 

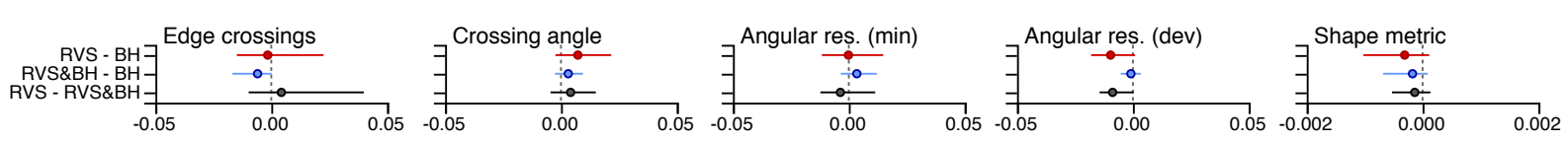

Figure 5: Bootstrap $99 . \overline{6} \%$ confidence interval (CI) of the effect sizes of the graph readability metrics. CIs are percentile bootstrap with 10,000 samples. CIs that touch zero indicate no statistically significant difference. Circles indicate effect size (sample mean difference). For a row (e.g. RVS - BH) a positive effect size indicates that the first algorithm (e.g. RVS) performs better. Note the differences in x-axis scales.
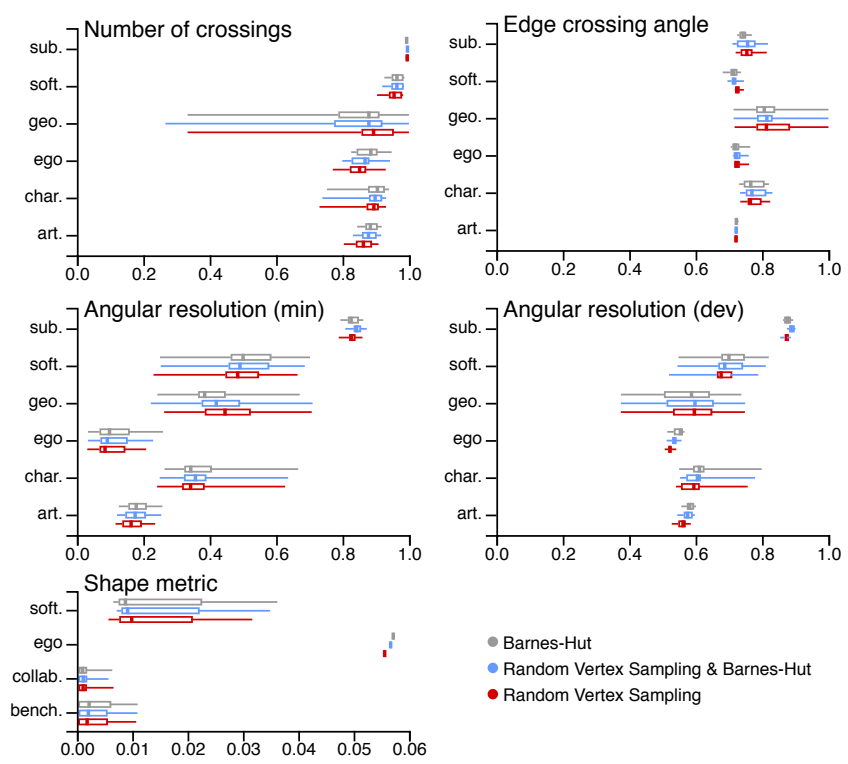

- Barnes-Hut

- Random Vertex Sampling \& Barnes-Hut - Random Vertex Sampling

Figure 6: Box plot of each graph class on each readability metric (minimum, 1st quartile, median, 3rd quartile, maximum).

we lack statistical evidence to say whether one repulsive-force algorithm produces better layouts than the others; they appear to produce comparable results, as measured by these readability metrics. Note that even if the effects were statistically significant, the effect sizes would be small. For example, a mean difference in angular resolution (dev) of 0.005 corresponds to a difference in mean deviation from the ideal angle of about 0.35 degrees.

Figure 6 explores the relationship between graph class, layout algorithm, and readability metric. The figure plots the box plot of each readability metric for each graph class using each layout algorithm. As shown in Table 2, the graph classes have differences in the number of vertices, the number of edges, ratio of components to vertices, and density. This appears to reveal some trends. First, we see that there can be a big difference in readability metric values between graph classes; especially for the angular resolution metrics. Second, we also see that there are some graph classes where one layout algorithm has a slight, consistent improvement over the others. For example, RVS performs the same or better than BH on geometric graphs across all readability metrics, but the opposite is true for artificial graphs. However, similar to the effect sizes above, the median differences tend to be small, so they may not represent a layout that is meaningfully better. Nonetheless, sometimes the interquartile range of readability metrics from different layouts on the same graph can be large (see the supplementary data), indicating that in some cases users may wish to generate multiple layouts using different initial positions and then choose the best one, regardless of the layout algorithm used.

\subsection{Visual Comparison}

Figure 7 shows example graph layouts created with RVS, BH, and RVS\&BH. Most graphs appear very similar in all layout algorithms. For some graphs, RVS creates a more compact layout, such as Miserables and eva. With RVS, graphs with multiple components can appear a little more chaotic (e.g. Netscience), whereas RVS and BH tend to be similar on geometric graphs (e.g. 4d hypercube and plat1919). RVS\&BH often has a global layout similar to RVS, but with better local symmetry. Figure 11 in Appendix A shows additional graph layouts.

\section{Convergence Rates}

Although using stress as a graph layout quality metric can have problems (see the discussion in subsection 6.1), it is widely used and regarded as an important metric. (Stress measures the difference between the embedded distance and geodesic distance of the vertices.) We are also interested in understanding whether using random vertex sampling (RVS) or the combination algorithm instead of Barnes-Hut (BH) affects the convergence rate of the layout algorithm. Therefore, this section presents an analysis of stress for each algorithm over 300 iterations on six graphs. These graphs are from the 109 experimental graphs, and they were chosen because they have distinct topologies and sizes, but only one component. The stress and layout time are averaged after each iteration across 40 runs of each algorithm. In most cases, RVS and RVS\&BH converge faster and achieve lower overall stress than BH (see Figure 8). This implies that BH's increased accuracy does not help it converge faster. In most cases, RVS and RVS\&BH converge to the same values, but in some cases RVS\&BH has a noticeable improvement at the end when BH refines the layout (e.g. Miserables).

\section{Discussion and Future Work}

Although all layout algorithms appear to produce good layouts on small graphs, when comparing these layouts to those produced by other algorithms (e.g. see MARS [KHKS12], Hu's algorithm [Hu05], and the sparse stress model [OKB16]), the other algorithms appear to produce better layouts on a few of the large graphs (e.g. finance256, dwt_1005, and plat1919) than the versions of the RVS, BH, and RVS\&BH algorithms used in this paper. This could be because the gravitational force used in these experiments prohibits vertices from finding better positions. (Gravity primarily benefits graphs with multiple components [FLM95] and is not used in some other layout algorithms.) The RVS, BH, and RVS\&BH layout algorithms could achieve 
R. Gove / Random Sampling Force Calculation
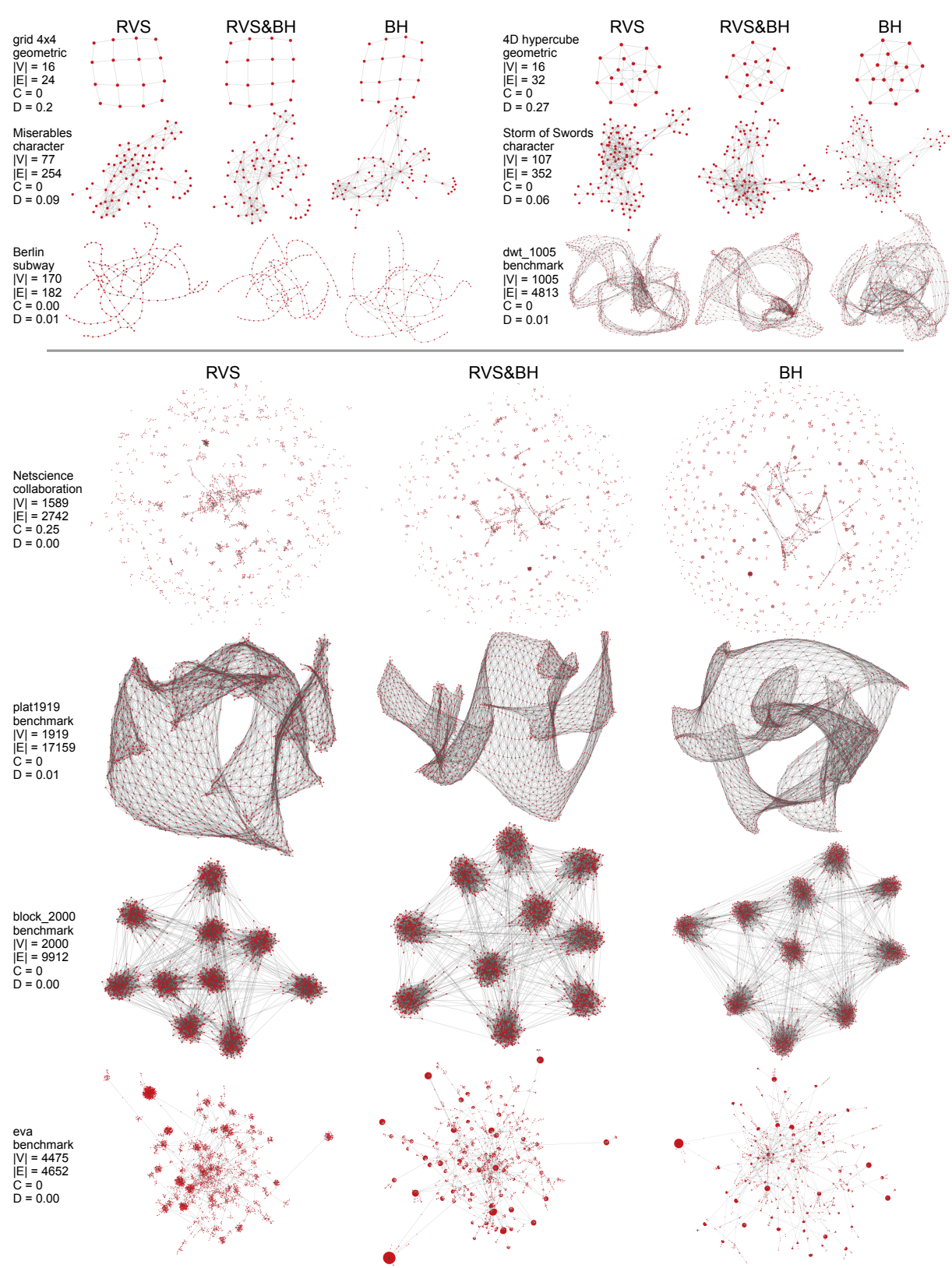

Figure 7: Comparison of random vertex sampling (RVS), Barnes-Hut (BH), and RVS combined with BH (RVS\&BH) graph layouts.

better layout quality on some graphs with different parameters. For example, Figure 9 shows the dwt_1005 and plat1919 graphs after 300 iterations of the RVS layout algorithm with alpha https://www.overleaf.com/project/5c0c13833b5200372464d136decay $\alpha_{d}=0$ and no gravitational force, which appears very similar to layouts produced by other algorithms [OKB16, KRM*17]. However, the goal of this paper is not to tune parameters for specific graph types, but rather to show that RVS has comparable layout quality as $\mathrm{BH}$ while achieving substantially faster performance.

Some layout algorithms use spring-electric forces with constraints, such as constraining vertices to rectangular regions [HM96] or orthogonal layouts [KDMW16]. Using the Les
Miserables graph, Figure 10 shows that RVS can be composed with many types of forces and constraints: Vertices repel each other using RVS as in the experiments above; edges attract vertices, but the force is stronger for vertices within the same group; constraints confine vertices to rectangular regions based on the book volume that introduced each character; and each rectangular region has a gravitational force that draws the vertices of that region toward its center. Future work should more closely compare quality and speed when using brute force, $\mathrm{BH}$, and RVS force-calculation algorithms to combine forces or generate constrained layouts.

This research finds that shape-based metrics can have a very narrow range of values, which is similar to Nguyen et al.'s find- 

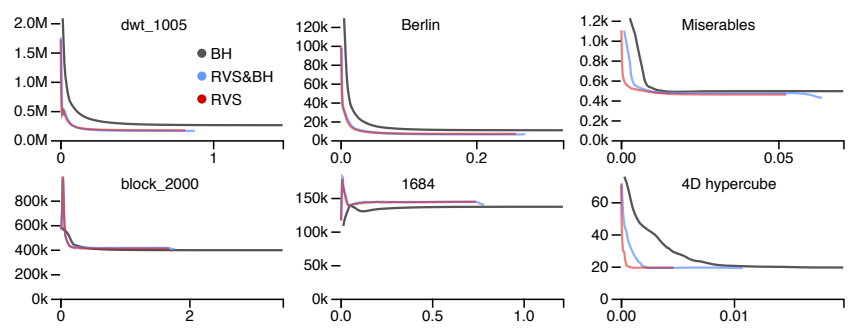

Figure 8: Stress (y axis) over time (x axis, seconds) of six graphs for the RVS, BH, and RVS\&BH combination layout algorithms for 300 iterations. Averaged over 40 runs of each algorithm.
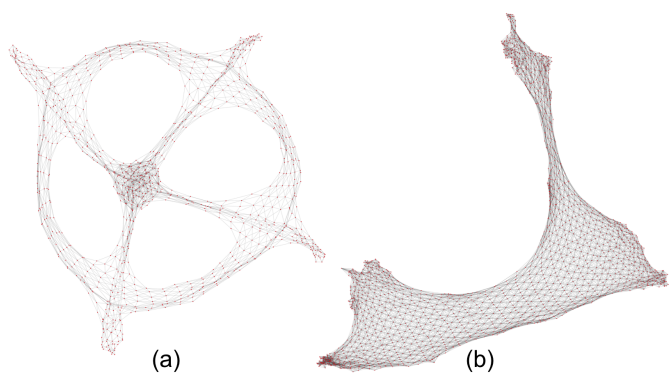

Figure 9: The dwt_1005 (a) and plat1919 (b) graphs using the RVS layout with alpha decay $\alpha_{d}=0$ and no gravitational force.

ings [NHE17]. However, unlike their work, this research also finds that the range for a shape-based metric can be very narrow even when using the dNNG proximity graph. For the large graphs tested here, the shape metric was always less than 0.06 . Such a narrow range makes it difficult to determine whether a change in the metric has a meaningful impact on the layout quality; especially because the large graph layouts shown in Figure 7 have a low shape metric but subjectively appear to do a good job of visualizing each graph's overall shape, which is the intended goal of the shape-based metric. Further research is needed to better understand the appropriate use of shape metrics, and to determine whether a different proximity graph would yield a more useful range of values.

Although RVS is faster than $\mathrm{BH}$, runtime performance can improve further through parallelization, since RVS is embarrassingly parallelizable. Future work should also investigate applying RVS to other domains, such as computing forces in $n$-body physics simulations, or speeding up t-SNE [vdM14].

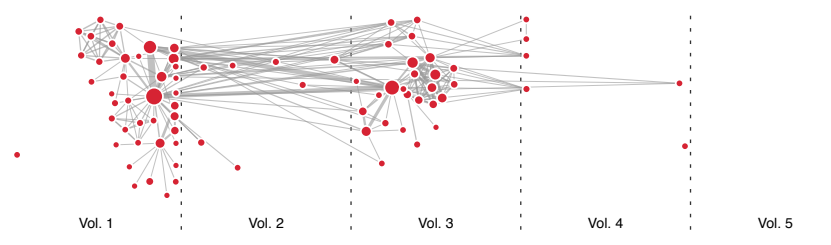

Figure 10: Visualization of the Les Miserables data set by composing spring-electric forces with a gravitational force for each Volume and rectangular constraints on $x, y$ vertex coordinates.

\section{Conclusion}

This paper presents the random vertex sampling (RVS) method for computing repulsive forces, and applies this method to computing graph layouts. This results in a layout algorithm with $O(|V|+|E|)$ running time per iteration and $O\left(|V|^{3 / 4}\right)$ auxiliary space, an asymptotic improvement over existing force-directed algorithms. In practice, RVS runs up to a 6.1 times faster (mean is 2.9 times faster) than the Barnes-Hut (BH) based graph layout algorithm while also producing comparable graph layout quality as $\mathrm{BH}$. This paper also proposes a combination algorithm that initially uses RVS to generate a layout and then uses $\mathrm{BH}$ to refine the layout. This combination algorithm runs substantially faster than $\mathrm{BH}$, but produces layouts that are more symmetric than RVS.

The RVS algorithm retains the conceptual simplicity of the typical force-directed algorithms without adding dependencies on complicated mathematical operations or data structures. This could aid adoption by practitioners. A version of this algorithm has already been adopted in a graph analysis tool [Gov16]. The RVS technique could replace the BH or FMM techniques used in graph layout algorithms [Hu05, JVHB14, QE00, HJ05b], particle simulations, or other algorithms such as t-SNE [vdM14].

\section{Acknowledgements}

Thanks to C. Clark, N. Danneman, J. Moore, O. Olmedo, M. Santoro, S. Stanley, C. Twardy, and M. Wakid for helpful discussion.

\section{References}

[ADlM15] Arleo A., Didimo W., Liotta G., Montecchiani F.: A Million Edge Drawing for a Fistful of Dollars. In International Symposium on Graph Drawing and Network Visualization (2015), pp. 44-51. 1,2

[agPS98] Aluru S., Gustafson J., Prabhu G., Sevilgen F. E.: Distribution-independent hierarchical algorithms for the n-body problem. The Journal of Supercomuting 12, 4 (1998), 303-323. 2, 3

[AMA07] Archambault D., Munzner T., Auber D.: TopoLayout: Multilevel graph layout by topological features. IEEE Transactions on Visualization and Computer Graphics 13, 2 (2007), 305-317. 2, 3, 6

[BA99] BARABÁsi A.-L., ALBERT R.: Emergence of scaling in random networks. science 286, 5439 (1999), 509-512. 6

[Bel] Belmonte N. G.: JavaScript InfoVis Toolkit. URL: http:// philogb.github.io/jit/. 1,2

[BH86] BARNES J., HUT P.: A hierarchical $\mathrm{O}(\mathrm{N} \log \mathrm{N})$ force calculation algorithm. Nature 324 (1986), 446-449. 2, 3

[BH09] Bostock M., Heer J.: Protovis: A graphical toolkit for visualization. IEEE Transactions on Visualization and Computer Graphics 15, 6 (2009), 1121-1128. 1, 2

[BhJ09] Bastian M., Heymann S., Jacomy M.: Gephi: An Open Source Software for Exploring and Manipulating Networks. In International AAAI Conference on Weblogs and Social Media (2009), pp. 361362. 1,2

[BOH11] Bostock M., Ogievetsky V., Heer J.: Data-Driven Documents. IEEE Transactions on Visualization and Computer Graphics 17, 12 (2011), 2301-2309. 1, 2, 3

[BP06] BRandes U., PICH C.: Eigensolver Methods for Progressive Multidimensional Scaling of Large Data. In International Symposium on Graph Drawing (2006), pp. 42-53. 1, 3, 6 
[Car14] CARLISLE R.: Arguing for a negligible effect. American Journal of Political Science 58, 4 (2014), 1083-1091. 7

[Cha96] Chalmers M.: A linear iteration time layout algorithm for visualising high-dimensional data. In Proceedings of Seventh Annual IEEE Visualization '96 (1996), pp. 127-132. 3, 4

[CK95] Callahan P. B., Kosaraju S. R.: A decomposition of multidimensional point sets with applications to k-nearest-neighbors and nbody potential fields. Journal of the ACM 42, 1 (1995), 67-90. 2, 3

[ÇMIBR06] ÇIVRIL A., Magdon-Ismail M., BoceK-Rivele E.: SSDE: Fast Graph Drawing Using Sampled Spectral Distance Embedding. In International Symposium on Graph Drawing (2006), pp. 30-41. $1,3,6$

[Dar00] DARVE E.: The fast multipole method: Numerical implementation. Journal of Computational Physics 160, 1 (2000), 195-240. 2

[DH96] DAVIDSON R., HAREL D.: Drawing graphs nicely using simulated annealing. ACM Transactions on Graphics 15, 4 (1996), 301-331. 5

[DH11] DaVIS T., HU Y.: The University of Florida Sparse Matrix Collection. ACM Transactions on Mathematical Software 38, 1 (2011), 1:1$1: 25.6$

[DLF*09] DWYER T., LEE B., FISHER D., QUINN K. I., ISENBERG P., Robertson G., North C.: A comparison of user-generated and automatic graph layouts. IEEE Transactions on Visualization and Computer Graphics 15, 6 (2009), 961-968. arXiv: 1408 .4626. 1, 2

[Dra16] DRAGICEVIC P.: Fair statistical communication in hci. In Modern Statistical Methods for HCI, Robertson J., Kaptein M., (Eds.). Springer, 2016, ch. 13, pp. 291-330. 7

[DRSM15] Dunne C., Ross S. I., Shneiderman B., Martino M.: Readability metric feedback for aiding node-link visualization designers. IBM Journal of Research and Development 59, 2/3 (2015), 14:1-14:16. 7

[Ead84] EADES P.: A heuristic for graph drawing. Congressus numerantium 42 (1984), 149-160. 2, 3, 5

[EHNK17] EAdes P., Hong S.-H., NGuYen A., Klein K.: ShapeBased Quality Metrics for Large Graph Visualization. Journal of Graph Algorithms and Applications 21, 1 (2017), 29-53. URL: http:// jgaa. info/getPaper?id=405. 7

[ENH17] EAdes P., NGUYen Q., Hong S.-H.: Drawing Big Graphs using Spectral Sparsification. In International Symposium on Graph Drawing and Network Visualization (2017), pp. 272-286. URL: http: //arxiv.org/abs/1708.08659, arXiv:1708.08659.3

[FLM95] FRICK A., Ludwig A., MEhldAu H.: A fast adaptive layout algorithm for undirected graphs. In International Symposium on Graph Drawing (1995), pp. 388-403. 5, 8

[FR91] Fruchterman T. M., Reingold E. M.: Graph drawing by force-directed placement. Software: Practice and Experience 21, 11 (1991), 1129-1164. arXiv: 91/111129âĂş36. 1, 2, 3, 5

[Gab15] GABAsOva E.: The Star Wars social network, 2015. URL: https://github.com/evelinag/ StarWars-social-network. 6

[GK02] Gajer P., Kobourov S. G.: GRIP: Graph dRawing with intelligent placement. J. Graph Algorithms Appl. 6, 3 (2002), 203-224. 1, 3,6

[GKN04] GANSNER E. R., Koren Y., NORTh S. C.: Graph drawing by stress majorization. In International Symposium on Graph Drawing (2004), pp. 239-250. 7

[GN99] GANSNER E. R., North S. C.: An open graph visualization system and its applications to software engineering. Software Practice and Experience 30, 11 (1999), 1203-1233. 1, 2, 7

[Gov16] Gove R.: V3SPA: A visual analysis, exploration, and diffing tool for SELinux and SEAndroid security policies. In IEEE Symposium on Visualization for Cyber Security (VizSec) (2016), pp. 1-8. 10
[Gov18a] Gove R.: Greadability.js, 2018. URL: https://github. com/rpgove/greadability. 7

[Gov18b] Gove R.: It pays to be lazy: Reusing force approximations to compute better graph layouts faster. In 11th Forum Media Technology (2018), pp. 43-51. 2, 7

[Gre87] Greengard L. F.: The Rapid Evaluation of Potential Fields in Particle Systems. PhD thesis, Yale University, New Haven, CT, USA, 1987. 2, 3

[HA15] Hinge A., Auber D.: Distributed graph layout with spark. In 19th Conference on Information Visualization (iV) (2015), pp. 271-276. 2,3

[HCL05] Heer J., CARd S. K., LANDAY J. A.: Prefuse: a toolkit for interactive information visualization. In Proceedings of the SIGCHI Conference on Human Factors in Computing Systems (2005), pp. 421-430. 1,2

[Hee08] HeER J.: Flare: Data Visualization for the Web, 2008. 1, 2

[HJ04] HACHUL S., JÜNGER M.: Drawing large graphs with a potentialfield-based multilevel algorithm. In International Symposium on Graph Drawing (2004), pp. 285-295. 2

[HJ05a] HACHUl S., JÜNGER M.: An Experimental Comparison of Fast Algorithms for Drawing General Large Graphs. In International Symposium on Graph Drawing (2005), pp. 235-250. 6

[HJ05b] Hachul S., JÜNGER M.: Drawing Large Graphs with a Potential-Field-Based Multilevel Algorithm. In Lecture Notes in Computer Science, Pach J., (Ed.). Springer-Verlag, Berlin, 2005, ch. Graph Drawing 2004, pp. 285-295. 1, 2, 3, 10

[HJ07] HaChul S., JÜNGER M.: Large-Graph Layout Algorithms at Work: An Experimental Study. Journal of Graph Algorithms and Applications JGAA 11, 2 (2007), 345-369. 3, 6

[HK02] HAREL D., KorEN Y.: Graph Drawing by High-Dimensional Embedding. In International symposium on graph drawing (Heidelberg, 2002), pp. 207-219. 3, 6

[HM96] He W., MarriotT K.: Constrained graph layout. In International Symposium on Graph Drawing (1996), pp. 217-232. 9

[HMF*17] Holanda A. J., Matias M., Ferreira S. M. S. P., Benevides G. M. L., KinOUCHI O.: Character Networks and Book Genre Classification. ArXiv e-prints (2017), arXiv: 1704.08197 .6

[HS95] HAREL D., SARDAS M.: Randomized graph drawing with heavy-duty preprocessing. Journal of Visual Languages and Computing 6, 3 (1995), 233-253. 5

[HSS08] Hagberg A. A., Schult D. A., Swart P. J.: Exploring network structure, dynamics, and function using NetworkX. In Proc. SciPy (2008), pp. 11-15. 6

[Hu05] HU Y.: Efficient, High-Quality Force-Directed Graph Drawing. Mathematica Journal 10, 1 (2005), 37-71. 1, 2, 6, 8, 10

[Hua07] HUANG W.: Using eye tracking to investigate graph layout effects. In Asia-Pacific Symposium on Visualisation 2007, APVIS 2007, Proceedings (2007), pp. 97-100. 1, 2

[JVHB14] JaCOMY M., Venturini T., Heymann S., Bastian M.: ForceAtlas2, a continuous graph layout algorithm for handy network visualization designed for the Gephi software. PloS one 9, 6 (2014). 1, 2, 10

[KCH02] Koren Y., CARMel L., Harel D.: ACE: A fast multiscale eigenvectors computation for drawing huge graphs. In Information Visualization (2002), pp. 137-144. 1, 3, 6

[KDMW16] KiefFer S., DWyer T., MARriot K., Wybrow M.: Hola: Human-like orthogonal network layout. IEEE Transactions on Visualization and Computer Graphics 1, 22 (2016), 349-358. 9

[KHKS12] Khoury M., Hu Y., Krishnan S., ScheidegGer C.: Drawing Large Graphs by Low-Rank Stress Majorization. Computer Graphics Forum 31, 3pt1 (2012), 975-984. 2, 6, 8 
[Knu94] KNUTH D. E.: The Stanford GraphBase: A Platform for Combinatorial Computing, 1st ed. ACM Press, 1994. 6

[Kob16] Ковоurov S. G.: Force-Directed Drawing Algorithms. In Handbook of Graph Drawing and Visualization, Tamassia R., (Ed.), 1 ed. Chapman and Hall/CRC, Boca Raton, 2016, ch. 12, pp. 383-408. arXiv: $1201.3011 .1,2,3$

[KPS14] Kobourov S. G., Pupyrev S., Saket B.: Are Crossings Important for Drawing Large Graphs? In International Symposium on Graph Drawing (2014), pp. 234-245. 7

[KPS17] Kobourov S., Pupyrev S., Simonetto P.: GMap Datasets, 2017. URL: http: / / gmap. cs . arizona. edu/datasets. 6

[KRM*17] Kruiger J. F., RAuber P. E., Martins R. M., KerRen A., Kobourov S., Telea A. C.: Graph Layouts by t-SNE. Computer Graphics Forum 36, 3 (2017), 283-294. 3, 6, 7, 9

[LKF07] Leskovec J., Kleinberg J., Faloutsos C.: Graph Evolution: Densification and Shrinking Diameters. ACM Trans. Knowl. Discov. Data 1, 1 (2007). 6

[LWZ15] LIPP F., WOLFF A., ZINK J.: Faster force-directed graph drawing with the well-separated pair decomposition. In International Symposium on Graph Drawing and Network Visualization (2015), pp. $52-59.1,2$

[LWZ16] LIPP F., WolfF A., ZINK J.: Faster Force-Directed Graph Drawing with the Well-Separated Pair Decomposition. Algorithms 9, 3 (2016), 53. 2

[ML12] McAuley J., Leskovec J.: Learning to Discover Social Circles in Ego Networks. In Proceedings of the 25th International Conference on Neural Information Processing Systems - Volume 1 (2012), NIPS'12, Curran Associates Inc., pp. 539-547. URL: http://dl. acm.org/citation. cfm?id=2999134.2999195. 6

[MMM08] Muelder C., MA K.-L., Member S.: Rapid Graph Layout Using Space Filling Curves. IEEE Transactions on Visualization and Computer Graphics 14, 6 (2008), 1301-1308. 6

[MRC03] MORrison A., Ross G., Chalmers M.: Fast multidimensional scaling through sampling, springs and interpolation. Information Visualization 2, 1 (2003), 68-77. 3

[New01] NEWMAN M. E. J.: The structure of scientific collaboration networks. Proceedings of the National Academy of Sciences 98, 2 (2001), 404-409. 6

[New06] NEwMAN M. E. J.: Finding community structure in networks using the eigenvectors of matrices. Physical Review E 74, 3 (2006), 036104. 6

[NHE17] NGUyen Q. H., Hong S. H., EAdes P.: DNNG: Quality metrics and layout for neighbourhood faithfulness. In IEEE Pacific Visualization Symposium (2017), pp. 290-294. 7, 10

[NHEM17] Nguyen Q. H., Hong S. H., Eades P., Meidiana A.: Proxy graph: Visual quality metrics of big graph sampling. IEEE Transactions on Visualization and Computer Graphics 23, 6 (2017), 16001611. 3

[OKB16] Ortmann M., Klimenta M., Brandes U.: A sparse stress model. In International Symposium on Graph Drawing and Network Visualization (2016), pp. 18-32. arXiv: 1608 .08909. 6, 8, 9

[PSD09] Pohl M., SchmitT M., Dienl S.: Comparing the Readability of Graph Layouts using Eyetracking and Task-oriented Analysis. In Computational Aesthetics in Graphics, Visualization, and Imaging (2009), pp. 49-56. 1, 2

[QE00] QUIGLEY A., EADES P.: FADE: Graph drawing, clustering, and visual abstraction. In International Symposium on Graph Drawing (2000), pp. 197-210. 1, 2, 3, 6, 10

[RA15] Rossi R. A., Ahmed N. K.: The network data repository with interactive graph analytics and visualization. In Proceedings of the Twenty-Ninth AAAI Conference on Artificial Intelligence (2015). URL: http://networkrepository.com. 6
[RC05] RAFIEI D., CURIAL S.: Effectively Visualizing Large Networks Through Sampling. In Visualization (2005), pp. 375-382. 3

[RKBB12] Roth C., KAng S. M., BAtTy M., BARThelemy M.: A long-time limit for world subway networks. Journal of the Royal Society Interface (2012). 6

[Tut63] TutTE W. T.: How to draw a graph. Proceedings of the London Mathematical Society 3, 1 (1963), 743-767. 2, 5

[VA10] Visscher P., APALKov D.: Simple recursive implementation of fast multipole method. Journal of Magnetism and Magnetic Materials 322, 2 (2010), 275-281. 2

[vB02] ŠUBELJ L., BAJEC M.: Clustering assortativity, communities and functional modules in real-world networks. ArXiv e-prints (2012-02). arXiv: 1202.3188 .6

[vB11] ك̌UBelJ L., BAJEC M.: Community structure of complex software systems: Analysis and applications. Physica A: Statistical Mechanics and its Applications 390, 16 (2011), 2968-2975. 6

[vB12] ŠUbelu L., BAJEC M.: Software systems through complex networks science: Review, analysis and applications. In Proceedings of KDD Workshop on Software Mining (2012), pp. 9-16. 6

[vBB13] Šubelj L., Bajec M., Blagus N.: Group extraction for real-world networks: The case of communities, modules, and hubs and spokes. In Proceedings of International Conference on Network Science (2013), pp. 152-153. 6

[vdM14] VAN DER MAATEN L.: Accelerating t-sne using tree-based algorithms. The Journal of Machine Learning Research 15, 1 (2014), 3221-3245. 10

[VKS*11] Von Landesberger T., KuiJPer A., Schreck T., Kohlhammer J., van WiJK J. J., Fekete J. D., Fellner D. W.: Visual analysis of large graphs: state-of-the-art and future research challenges. Computer Graphics Forum 30, 6 (2011), 1719-1749. 2, 3, 5

[VR08] VAN HAM F., Rogowitz B. E.: Perceptual organization in user-generated graph layouts. IEEE Transactions on Visualization and Computer Graphics 14, 6 (2008), 1333-1339. 1, 2

[vvBB14] Šubelu L., ŽItnik S., Blagus N., Bajec M.: Node mixing and group structure of complex software networks. Advances in Complex Systems 17, 7 (2014), 1450022. 6

[Wal00] WALSHAW C.: The graph partitioning archive, 2000. URL: http: / / chriswalshaw.co.uk/partition/. 6

[WCA*17] Wu Y., CaO N., Archambault D., Shen Q., Qu H., CuI W.: Evaluation of Graph Sampling: A Visualization Perspective. IEEE Transactions on Visualization and Computer Graphics 23, 1 (2017), 401-410. 3

[Won17] WONGSUPHASAWAT K.: Increasing the impact of visualization research, $2017 . \quad$ URL: https://www.slideshare.net/kristw/ increasing-the-impact-of-visualization-research.

[WS98] WatTs D. J., Strogatz S. H.: Collective dynamics of 'smallworld' networks. Nature 393, 6684 (1998), 440-442. 6

[Zha15] ZHANG F.: A Visual and Statistical Benchmark for Graph Sampling Methods. In Exploring Graphs at Scale Workshop (2015). 3

[ZZC*17] Zhang F., Zhang S., Chung P., Hugh W., LinkAn M., EDWARD B. J., II S.: A Visual Evaluation Study of Graph Sampling Techniques. In Visualization and Data Analysis (2017), pp. 110-117. 3

Appendix A: Additional Graph Drawing Comparisons 
R. Gove / Random Sampling Force Calculation
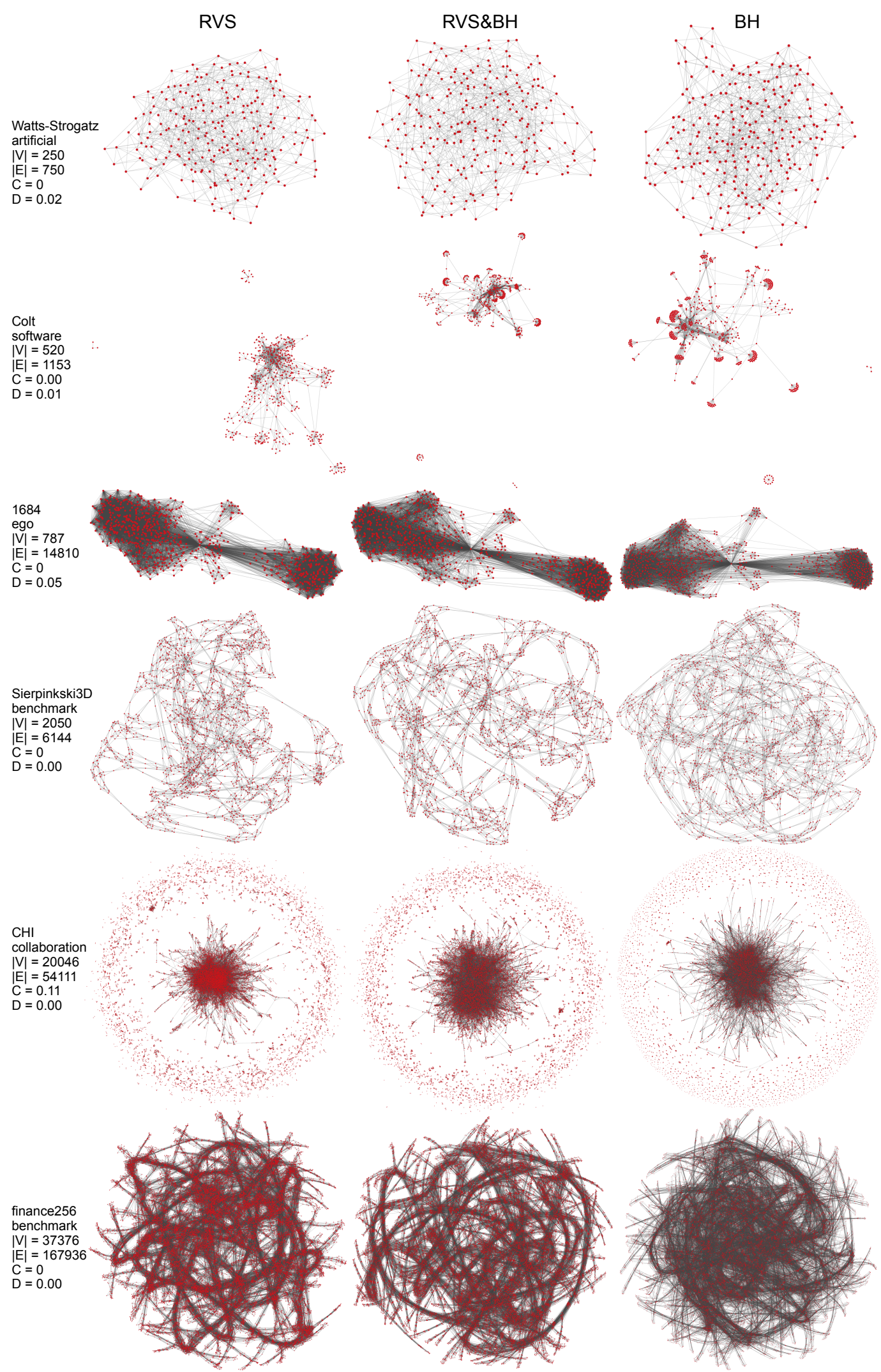

Figure 11: Comparison of random vertex sampling (RVS), Barnes-Hut (BH), and RVS combined with BH (RVS\&BH) graph layouts. 